\title{
Erratum to: Evolution of gut microbiota composition from birth to 24 weeks in the INFANTMET Cohort
}

Cian J. Hill1 ${ }^{1,2}$, Denise B. Lynch ${ }^{1,2}$, Kiera Murphy ${ }^{1,2,3}$, Marynka Ulaszewska ${ }^{5}$, lan B. Jeffery', Carol Anne O'Shea ${ }^{4}$, Claire Watkins ${ }^{3}$, Eugene Dempsey ${ }^{4}$, Fulvio Mattivi ${ }^{5}$, Kieran Tuohy ${ }^{5}$, R. Paul Ross ${ }^{1,2}$, C. Anthony Ryan ${ }^{2,4}$, Paul W. O'Toole ${ }^{1,2}$ and Catherine Stanton ${ }^{2,3^{*}}$

\section{Erratum}

Following publication of this article [1], it has come to our attention that the name of the author Kieran Tuohy's name was captured incorrectly as "Touhy" and instead should be Kieran Tuohy. The original article has also been corrected.

\section{Author details}

${ }^{1}$ School of Microbiology, University College Cork, Cork, Ireland. ${ }^{2}$ APC Microbiome Institute, University College Cork, Cork, Ireland. ${ }^{3}$ Teagasc Moorepark Food Research Centre, Fermoy, Co., Cork, Ireland. ${ }^{4}$ Department of Neonatology, Cork University Maternity Hospital, Cork, Ireland. ${ }^{5}$ Food Quality and Nutrition Department, Research and Innovation Centre, Fondazione Edmund Mach, San Michele All'adige, Italy.

Received: 31 January 2017 Accepted: 31 January 2017

Published online: 14 February 2017

\section{Reference}

1. Hill JC, Lynch BD, Murphy K, Ulaszewska M, Jeffery BI, O'Shea CA, Watkins C, Dempsey E, Mattivi F, Tuohy K, Ross PR, Ryan CA, O' Toole WP, Stanton C. Evolution of gut microbiota composition from birth to 24 weeks in the INFANTMET Cohort. Microbiome. 2017;5:4. doi:10.1186/s40168-016-0213-y.

* Correspondence: Catherine.stanton@teagasc.ie

${ }^{2}$ APC Microbiome Institute, University College Cork, Cork, Ireland

${ }^{3}$ Teagasc Moorepark Food Research Centre, Fermoy, Co., Cork, Ireland 